\title{
DYNAMO IN THE PRESENCE OF \\ DIFFERENTIAL ROTATION
}

\author{
S. I. VAINSHTEIN \\ Sibizmiran, U.S.S.R.
}

It is well know that the 'dynamo' theory has a number of vetoes; e.g. axisymmetric, two-dimensional, central-symmetric, etc. dynamo are impossible. In principle, the problem is essentially three-dimensional in any coordinate system. This is the main difficulty of both the theory itself and its possible applications. In fact, one prefers to believe that, for example, a non-rigid body-rotating star or convection in the Earth's nucleus possesses axis symmetry. However, due to the above vetoes one has to add finer effects (Coriolis strength, density, inhomogeneity) to create asymmetrical convection. On the other hand, the authors try to find the most simple movements with minimum deviations from axial symmetry. Thus, the Herzenberg's dynamo (Herzenberg, 1958) is realized by two rotating cylinders, axes of which are parallel to each other (see also Galaitis, 1973; Galaitis and Freinberg, 1974), the Lortz's dynamo-spiral movement (Lortz, 1968; Ponomarenko, 1973). Nevertheless, the mentioned vetoes possess a common feature, the assumption regarding the symmetry extends both to the movement and to the field. Hence, it makes sense to raise a question whether symmetric movements are able to generate an asymmetric field. A positive answer to this question, in particular, is given by Tverskoy's model (Tverskoy, 1966) - the toroidal vortex. The latter possesses axial symmetry. Nevertheless, the toroidal vortex is a complex motion; we will proceed along the path of a minimum simplification.

Is dynamo possible in differential rotation? This motion is the simplest and likely the most wide-spread in nature. Besides, is dynamo possible in two-dimensional motion of the differential rotation type, that is, in the cylindrical system

$$
v_{r}=v_{z}=0, \quad v_{\varphi} \neq 0, \quad v_{\varphi}=v_{\varphi}(r) ?
$$

On the face of it, the answer must be negative as Zeldovich's system (Zeldovich, 1956) exists which rules out a two-dimensional dynamo in the arbitrary (that is, not necessary two-dimensional) magnetic field. In this problem, however, the velocity is even one-dimensional as $\mathbf{v}$ depends only on $r$. However, the mentioned theory is proved for the unlimited conductive medium. If the conductive medium is situated at $r<R$, and at $r>R$ the medium will be unconductive (in particular, vacuum), then the veto can be removed and dynamo is possible. We will notice that no unlimited bodies exist in nature.

Let us explain why the veto is taken away from the two-dimensional dynamo in the presence of vacuum. From induction equation

$$
\frac{\partial \mathbf{H}}{\partial t}=\operatorname{rot}[\mathbf{v H}]+\nu_{m} \Delta \mathbf{H}
$$


equation for $H_{z}$ results in given geometry

$$
\frac{\partial H_{z}}{\partial t}+(\mathbf{\nabla}) H_{z}=\nu_{m} \Delta H_{z}
$$

In view of the fact that the behaviour $H_{z}$ is described by the heat conductivity equation and $H_{z} \rightarrow 0$ at $r \rightarrow \infty$ in the unlimited fluid, $H_{z} \rightarrow 0$ at $t \rightarrow \infty$, that is, $H_{z}$ is damping.

Then, assuming that $H_{z}=0$, it is easy to prove that the equation for the vectorpotential of removed field components has also the form of conductivity equation. Hence, both $H_{x}$ and $H_{y}$ are suppressed.

Now, let the vacuum be at $r>R$. Then, assuming that the permanence for media $\mu=I$, we will have the boundary conditions: field continuity, while the field in vacuum is potential, as well as

$$
\frac{1}{r} \cdot \frac{\partial H_{z}}{\partial \varphi}-\frac{\partial H_{\varphi}}{\partial z}=0
$$

on the boundary. Condition (3) corresponds to zero reduction of the current component, normal toward the boundary. Now, conductivity Equation (2) with boundary conditions (3) must yield no field $H_{z}$ damping! In general, all theorems of dynamo impossibility are proved when one of the field components separates from the other, that is, it behaves independently of them. At the same time, $H_{z}$ is related to $H_{\varphi}$ through boundary condition (3). It will be shown below that just this very fact takes away the veto from the one-dimensional dynamo.

It seems that one might object that the vacuum boundary conditions on the sky body boundary are not very topical. The Sun, for instance, is surrounded by a highly conductive corona which directly passes into the solar wind. Are vacuum boundary conditions topical here? However, $H_{z}$ field component does not separate from the other already in the presence of electric conductivity dependence upon $r$ (thus $\nu_{m}$ is axisymmetric too). In fact, the equation for $H_{z}$ has in this case the form:

$$
\frac{\partial H_{z}}{\partial t}+(\mathbf{v} \nabla) H_{z}=\nu_{m}\left(\frac{1}{r} \cdot \frac{\partial}{\partial r} r \frac{\partial H_{z}}{\partial r}+\frac{1}{r^{2}} \cdot \frac{\partial^{2} H_{z}}{\partial \varphi^{2}}\right)-\frac{\partial \nu_{m}}{\partial r} \cdot \frac{\partial H_{r}}{\partial z}
$$

It can be seen from (4) that to conclude on damping is impossible again. Field generation exists in case (4) too. The assumption of inhomogeneous $\nu_{m}(r)$ is naturally associated with the sky body boundary itself; in the particular case of vacuum $\nu_{m}$ it changes sharply, in vacuum $\nu_{m}=\infty$.

\section{One-Dimensional Problem Solution in Vacuum}

Let us express the field $v_{\varphi}(r)$ in this way (Vainshtein, 1975):

$$
\begin{array}{llll}
\text { region I: } & v_{\varphi}=\omega_{0} r & \text { at } & r<r_{0} ; \\
\text { region II: } & v_{\varphi}=0 & \text { at } & r>r_{0}, r<R .
\end{array}
$$

The rigid body rotation at $r<r_{0}$ simplifies the calculations, although it is not 
important, in principle, whether there is a jump in angular velocity $\omega(r)$ or if the transition is smooth. Then, the equations for $H_{r}, H_{\varphi}, H_{z}$ have the form:

$$
\begin{aligned}
& \frac{\partial H_{z}}{\partial t}=-\omega_{0} \frac{\partial H_{z}}{\partial \varphi}+\nu_{m} \Delta H_{z} ; \\
& \frac{\partial H_{r}}{\partial t}=-\omega_{0} \frac{\partial H_{r}}{\partial \varphi}+\nu_{m}\left(\Delta H_{r}-\frac{1}{r^{2}} H_{r}-2 \frac{1}{r^{2}} \frac{\partial H_{\varphi}}{\partial \varphi}\right) ; \\
& \frac{\partial H_{\varphi}}{\partial t}=-\omega_{0} \frac{\partial H_{\varphi}}{\partial \varphi}+r H_{r} \cdot \frac{\partial \omega_{0}}{\partial r}+\nu_{m}\left(\Delta H_{\varphi}-\frac{1}{r^{2}} H_{\varphi}+2 \frac{1}{r^{2}} \cdot \frac{\partial H_{r}}{\partial \varphi}\right)
\end{aligned}
$$

The solution of the system (6) is to be naturally sought in the form:

$$
H_{r, \varphi, z}=f_{r, \varphi, z}(r) \exp [E t+i(m \varphi+k z)] \text {. }
$$

Similarly to (5), introduce the function $f_{ \pm}=f_{r} \pm i f_{\varphi}$ and we obtain Bessel equations for regions I, II and vacuum (region III):

$$
\begin{aligned}
& \frac{\partial^{2} f_{z}}{\partial p^{2}}+\frac{1}{p} \cdot \frac{\partial f_{z}}{\partial p}-\left(1+\frac{m^{2}}{p^{2}}\right) f_{z}=0 ; \\
& \frac{\partial^{2} f_{ \pm}}{\partial p^{2}}+\frac{1}{p} \cdot \frac{\partial f_{ \pm}}{\partial p}-\left(1+\frac{(m \pm 1)^{2}}{p^{2}}\right) f_{ \pm}=0
\end{aligned}
$$

where in region I

$$
p=\beta r, \quad \beta=\sqrt{\left(E+\nu_{m} k^{2}+i m \omega_{0}\right) / \nu_{m}} ;
$$

in region II

$$
p=x r, \quad x=\sqrt{\left(E+\nu_{m} k^{2}\right) / \nu_{m}} ;
$$

in region III

$$
p=k r \text {. }
$$

We are looking for the solution of set (8) in the form:

region I:

$$
f_{z}=A I_{m}(p), \quad f_{ \pm}=B_{ \pm} I_{ \pm}(p) \text { (limited in zero) } ;
$$

region II:

$$
f_{z}=C I_{m}(p)+D K_{m}(p), \quad f_{ \pm}=L_{ \pm} I_{ \pm}(p)+M_{ \pm} K_{ \pm}(p) ;
$$

region III:

$$
f_{z}=F K_{m}(p), \quad f_{ \pm}=i F K_{ \pm}(p) \text { (disappearing in infinity) }
$$

where

$$
I_{ \pm}=I_{m \pm 1}, \quad K_{ \pm}=K_{m \pm 1} .
$$

In region III we have made use of condition $\operatorname{rot} \mathbf{H}=0$. 
The unification of solutions in the III regions yields a set of algebraic equations for coefficients.

The coupling condition is as follows: the continuity of all solutions and

$$
\begin{aligned}
& \left.\frac{\partial f_{z}}{\partial r}\right|_{I}=\left.\frac{\partial f_{z}}{\partial r}\right|_{I I} ; \\
& \left.\left(\frac{\partial f_{ \pm}}{\partial r} \pm \frac{i r_{0} \omega_{0}}{2 \nu_{m}}\left(f_{+}+f_{-}\right)\right)\right|_{I}=\left.\frac{\partial f_{ \pm}}{\partial r}\right|_{I I}
\end{aligned}
$$

Nine equations and 10 coefficients one can obtain. The Equation (10) is obtained by using the condition $\operatorname{div} \mathbf{H}=\mathbf{0}$ (condition (3) is derived from the written ones). In order to derive this equation, take the divergence from (6), and we obtain:

$$
E \gamma-i m \omega_{0} \gamma=\nu_{m} \Delta \gamma ; \quad \gamma=\operatorname{div} \mathbf{H} \text {. }
$$

Further, in order $\gamma \equiv 0$, it is sufficient $\gamma$ to be equal to zero on the boundary with vacuum (this results from uniqueness of solution (10) in given boundary conditions). Writing $\operatorname{div} \mathbf{H}=\mathbf{0}$ on the boundary of the regions II-III and taking into account the fact that the field is continuously crossing the boundary, it is easy to understand that $\partial H_{\varphi} / \partial \varphi, \partial H_{z} / \partial z$ are also continuous on the boundary; from this

$$
\left.\frac{\partial H_{r}}{\partial r}\right|_{\mathrm{II}}=\left.\frac{\partial H_{r}}{\partial r}\right|_{\mathrm{III}}
$$

Condition (II) is the last equation to be found. The determinant of the tenth order is presented in the form of a two (co-)factor product, one of which does not yield dynamo.

Let us assume that $\beta r_{0}, x r_{0}, k R \gg 1$.

Below we will see that this situation corresponds to the great Reynolds number $R_{m}=\omega_{0} r_{0}^{2} / \nu_{m} \gg 1$. Now $p \gg 1$ on the boundaries, and one can use asymptotes

$$
\begin{aligned}
I_{m}(p) & =(1 / 2 \pi p)^{1 / 2}[\exp (p)+\exp (-p-(m+1 / 2) \pi i)], \\
K_{m}(p) & =(\pi / 2 p)^{1 / 2} \cdot \exp (-p)
\end{aligned}
$$

The second factor of the determinant is simplified, considering that th $\varphi \approx \pm 1$, $\varphi=\beta r_{0}+i(\pi / 2)\left(m+\frac{1}{2}\right)$, which is fulfilled with exponential accuracy. In this case, the given factor is divided, in its turn, into two factors, one of which yields the equation

$$
\text { th } x \Delta r=\mp \frac{x}{\beta} ; \quad \Delta r=R-r_{0},
$$

which does not yield dynamo. To the second factor corresponds the equation:

$$
\text { th } x \Delta r=x \frac{k \mp \beta}{x^{2} \mp \beta k}
$$

(The upper sign corresponds to th $\varphi=+\mathrm{I})$ or in the non-dimensional form $(q=\varkappa \Delta r)$

$$
\text { th } q=\frac{D-\sqrt{q^{2}+i C}}{q^{2}-D \sqrt{q^{2}+i C}}
$$


If in (15) we assume $D=k \Delta r, C=m \omega_{0}(\Delta r)^{2} / \nu_{m}, \operatorname{Re}\left(q^{2}+i C\right)^{1 / 2}>0 . \omega_{0}=0$, that is, $C=0$ (absence of rotation, the trivial case), then (15) has no roots and one must use other factors of the determinant giving field damping. If $m=0$ (the purely axisymmetric case, that is both the velocity and $\bar{H}$ are axisymmetric), then $C=0$, so that it is tantamount to $\omega_{0}=0$ and the dynamo is impossible (theorem by S. I. Braginsky, 1964).

If $k=0$, that is $D=0$ (the purely two-dimensional case), then the equation is analogous to (13), only on the right side instead of $x / \beta$ there is $\beta / x$. This equation also has no growing solutions (Ya. B. Zeldovich theorem (Zeldovich, 1956)).

At $\Delta r \rightarrow 0$ we obtain the rotating cylinder as a rigid-body in vacuum. It is natural that the dynamo is impossible $(D=C=0)$. It can be seen therefore that the field will be essentially three-dimensional and will possess all the three components.

It can be easily seen that for the dynamo-solution, the root must lie in the region $\operatorname{Re}\left(q^{2}-k^{2}\right)>0$. Solution (15) should be looked for in C-D-plane by giving $q$. Assuming $q=1.00-i 0.50$, we obtain graphically $C=0.11, D=-0.55$.

It is not difficult to verify that the given root corresponds to accepted assumptions and yields dynamo. In fact $\chi \Delta r \approx \beta \Delta r \approx k \Delta r \approx 1$ is not in contradiction with the use of asymptotes (12), if $r_{0} \gg \Delta r$; the latter condition corresponds to $\mathrm{Rm}=$ $C r_{0}^{2} / m(\Delta r)^{2} \gg 1$, i.e. to the great Reynolds number. Further

$$
E=\left(\varkappa^{2}-k^{2}\right) \nu_{m}=\frac{\nu_{m}}{(\Delta r)^{2}}(0.47-i 1.00)
$$

By using the condition $C \approx \mathrm{I}$, we obtain $\operatorname{Re} E \sim m \omega_{0}$, which is quite natural.

\section{Discussion}

The considered example supports the following arguments: dynamo occurs in all the cases when it is impossible to prove the contrary, using some standard rules. It seems that this affirmation is unlikely to be proved, nevertheless, it is practically always helpful.

In order to be sure of the existence of the dynamo in a determined situation, the following procedure should be adopted:

(1) Write the induction equation in the natural curvilinear coordinate system.

(2) Verify whether one of the field components is not prevalent; in the affirmative, this would lead to damping and the dynamo is impossible, as the remaining components are sure to be damped (which can be proved by transition to the equation for vector-potential).

Theorems on the impossibility of the dynamo were proved in just this way.

The astonishing simplicity and symmetry of the model under consideration permits one to hope that, practically, the veto for the dynamo will be taken away for all the symmetric models. But then, more complex asymmetric motions will be the more so dynamo-instable. Thus we might suggest a thesis-assumption: all motions in nature are instable with respect to magnetic fluctuations. The dynamo problem can be, therefore, turned upside down by looking for such motions in real limited bodies without generation. 
Since the dynamo-solution has a character of instability in relation to the magnetic field fluctuations, the latter will be excited at least by thermodynamical fluctuations no matter whether it corresponds to observational data or not. Therefore one may ask why the solar cycle period $\approx 22$ years, when $\omega_{0} \sim 10^{-6} \mathrm{~s}^{-1}-$ i.e., the field growth time caused by differential rotation is less than one month - and why all the celestial bodies do not have a magnetic field, etc. On the other hand the model considered is similar to the model of a rotating galaxy; the increment $\sim r \partial \omega / \partial r \sim \omega$, therefore the field is growing in the time period of a galaxy rotation, i.e. rather rapidly. And finally, both the occurrence of the field and its strengthening on the solar surface (for instance sunspots) as well as its disappearance can be easily explained by differential rotation only, taking into account either the simplest dynamo, or 'antidynamo' (Vainshtein, 1973). The analysis of the most simple shift motions the differential rotation and the shift (Vainshtein, 1973), showed that even such simple motions could cause a reduction of the field's scale and later on its strengthening or effective destruction. That is why the idea of the 'frozen-in' is a very simplified picture. Taking into account the increasing contribution of electromagnetic forces with decreasing field scale one can come to the following dilemma:

(1) If the electroconductivity is not very high (e.g. turbulent) and if in spite of the scale reduction, the electromagnetic forces remain insignificant, then the idea of the 'frozen-in' field is very often inadequate.

(2) If the electromagnetic forces contribution is essential, then the idea of the 'frozen-in' field is valid, however, the field of velocities itself acquires a small scale structure and becomes complicated.

\section{References}

Braginskij, S. I.: 1964, Zh. Exper. Teoret. Fiz. 47, 1084.

Galaitis, A.: 1973, Magnitnaya Gidrodinamika 4, 12.

Galaitis, A. and Freinberg, Ya.: 1974, Magnitnaya Gidrodinamika, No. 1, 37.

Herzenberg, A.: 1958, Phil. Trans. Roy. Soc. A. $250,543$.

Lortz, D.: 1968, Plasma Phys. 10,, No. 11, 967.

Ponomarenko, Yu. B.: 1973, Prikladnaya Mehanika i Tehnicheckaya Fizika, No. 6, 47.

Tverskoy, B. A.: 1966, Geomagnetizm i Aeronomija 6, 11.

Vainshtein, S. I.: 1973, Zh. Exper. Teoret. Fiz. 65, 550.

Vainshtein, S. I.: 1975, Zh. Exper. Teoret. Fiz. 62, 997.

Zel'dovich, Ya. B.: 1956, Zh. Exper. Teoret. Fiz. 31, 154.

\section{DISCUSSION}

Gilman: Your magnetic field produced by the dynamo must be non-axisymmetric. What form does it take? In particular are there wave numbers in the directions perpendicular to the shear of rotation for which dynamo occurs at the lowest magnetic Reynolds number?

Vainshtein: There is dependence on $r, z$ and $\varphi$. The dependence on $z$ is proportional to $I^{i k z}$ where $k$ is the wave number in the direction $z$ the dependence on $\varphi$ is proportional to $I^{i m \varphi}, m=1,2, \ldots$; Reynolds number must be great.

Deinzer: Are these magnetic fields at all if there is an axisymmetric flow? I think they would be excluded by Cowling's theorem.

Vainshtein: Cowling's theorem is proved for the case when both magnetic field and velocity field are axisymmetric. In my case the magnetic field is not axisymmetric. 
Stix: I agree with Dr Weiss that the model presented here is not in conflict with Cowling's theorem. But there is another theorem that says that no toroidal motion can give rise to dynamo action. Rotation, no matter how differential, is such a toroidal motion. The theorem has recently been in an even more restrictive form by Busse who found that the magnetic Reynolds number based on the radial velocity component must exceed a critical value. So my question is whether the model contradicts this theorem. Another question is: What are your boundary conditions at infinity?

Vainshtein: One cannot prove that the dynamo mechanism cannot act if the differential rotation only is present and conductivity is inhomogeneous.

Stix: I was referring to the magnetic Reynolds number based on the radial velocity component, not to the one based on the differential rotation.

Vainshtein: At infinity the magnetic field tends to zero. The generation of magnetic field can be in the presence of differential rotation without radial motion. 